\title{
Risk Factors for Development of Paradoxical Response during Anti-Tuberculosis Treatment in HIV-Negative Patients with Pleural Tuberculosis
}

\author{
Jae Woo Jung, ${ }^{1}$ Jong Wook Shin, ${ }^{1}$ Jae Yeol Kim, ${ }^{1}$ In Won Park, ${ }^{1}$ \\ Byoung Whui Choi, ${ }^{1}$ Jae Seung Seo ${ }^{2}$ and Jae Chol Choi ${ }^{1}$ \\ ${ }^{1}$ Division of Pulmonary and Critical Care Medicine, Department of Internal Medicine, Chung-Ang University \\ School of Medicine, Seoul, Korea \\ ${ }^{2}$ Department of Radiology, Chung-Ang University School of Medicine, Seoul, Korea
}

\begin{abstract}
Paradoxical response $(P R)$ is the unusual expansion or new formation of a tuberculous lesion during anti-tuberculosis (TB) treatment. Pleural TB is the second most common form of extrapulmonary TB and has clinical importance because it occurs in a restricted space. Limited information is available for PR in HIV-negative patients with pleural TB. The aim of this study was to evaluate the clinical characteristics and risk factors of PR in HIV-negative patients with pleural TB. Patients diagnosed with pleural TB between 2003 and 2008 at Chung-Ang University Hospital and Yong-San Hospital, Seoul, South Korea were included. We evaluated the incidence and treatment outcome of PR in pleural TB, and compared baseline clinical characteristics and laboratory findings between TB patients with PR and those without PR. PR was present in $32(23 \%)$ of 139 patients after mean 51.1 days following initiation of treatment. Out of 32 patients, 18 patients needed additional treatment for symptom control. PR patients had a high incidence of adverse drug reaction such as drug skin reaction or liver function abnormality $(P<0.05)$. The risk factors for PR are younger age, high serum albumin level, low proportion of lymphocyte, and high proportion of PMN in pleural fluid $(P<0.05)$. PR is not an uncommon problem in HIV-negative pleural TB and half of these patients need additional treatment. Therefore, physicians must pay more attention for PR during the management of pleural TB in the expected patients to develop PR.
\end{abstract}

Keywords: tuberculosis; paradoxical response; pleurisy; risk factors; HIV seronegativity

Tohoku J. Exp. Med., 2011, 223 (3), 199-204. C 2011 Tohoku University Medical Press

Pleural tuberculosis (TB) is estimated to constitute approximately $5-30 \%$ of all disease caused by Mycobacterium tuberculosis (Seibert et al. 1991; Ferrer Sancho 1996). The current recommendation for pleural TB is the same as for pulmonary TB (Bass et al. 1994). However, despite documented therapeutic successes, some patients show deterioration during anti-TB therapy. This deterioration is called paradoxical response (PR), and it remains a clinical challenge.

PR is the unusual expansion or new formation of a tuberculous lesion during TB treatment (Smith 1987). PR is one phenomenon of the immune reconstitution inflammatory syndrome and has been extensively reported in HIVpositive patients receiving highly active antiretroviral therapy (HAART) (Wendel et al. 2001; Cheng et al. 2002; Navas et al. 2002). However, PR can also occur in HIVnegative patients, especially in extra-pulmonary TB including pleural TB (Cheng et al. 2002; Cheng et al. 2007). Clinically, PR may be confused with treatment failure, drug resistant TB, or other infection, especially in extra-pulmonary cases that have a low positive rate of pathogen culture (Baumann et al. 2007). However, only limited information is available on the clinical characteristics and risk factors for PR of pleural TB in HIV-negative patients (Al-Majed 1996; Kumar and Vasu 2001; Choi et al. 2002).

The aim of this study was to estimate the incidence and clinical course of PR in HIV-negative patients with pleural TB and to determine the parameters for predicting PR in HIV-negative patients with pleural TB.

Methods
Study design and patients
Patients diagnosed with pleural TB between January 2003 and
December 2008 at Chung-Ang University Hospital and Yong-San
Hospital were included in this retrospective study. We excluded
patients who had pleural TB with HIV infection and also those
younger than 15 years of age. Anti-TB medications including isonia-
zid, rifampicin, ethambutol and pyrazinamide were administered. We

Received January 5, 2011; revision accepted for publication February 7, 2011. doi: 10.1620/tjem.223.199

Correspondence : Jae Chol Choi, M.D., Ph.D., Division of Pulmonary and Critical Care Medicine, Department of Medicine, Chung-Ang

University Young San Hospital, Han Gang Ro 3 Ga, Young San-ku, Seoul 140-757, Republic of Korea.

e-mail: medics27@ cau.ac.kr 
evaluated the incidence, clinical course, and treatment outcomes of PR in pleural TB. Demographic findings, adverse drug reactions, white blood cell (WBC) count, and pleural fluid parameters, including differential cell count, lactate dehydrogenase $(\mathrm{LDH})$, total proteins, and adenosine deaminase (ADA) levels, were compared between patients with PR and patients without PR when available. The Institutional Review Board of Yong-San Hospital, Seoul, Korea, and Chung-Ang University Hospital, Seoul, Korea, approved this study.

\section{Diagnostic criteria of pleural TB and $P R$}

The diagnosis of pleural TB depended on 1) the demonstration of acid-fast bacilli (AFB) or the growth of $M$. tuberculosis in pleural fluid or pleural biopsy specimens; 2) the presence of classical caseating granulomas in the pleural tissue and a successful response to antiTB therapy; or 3) a lymphocyte-predominant exudative effusion with ADA levels ( $>40 \mathrm{I} \mathrm{U/L)} \mathrm{and} \mathrm{a} \mathrm{successful} \mathrm{response} \mathrm{to} \mathrm{anti-TB} \mathrm{therapy}$ (Gopi et al. 2007). The positive predictive value of lymphocyte predominant exudates with ADA levels (> 40I U/L) is $100 \%$ in the Korean population (Kim et al. 2006).

PR was defined as the worsening of radiological findings following the initiation of appropriate anti-TB treatment in the absence of evidence of disease relapse or the presence of another diagnosis (Breen et al. 2004). Whom onset of paradoxical response occurs within 14 days after initiation of anti-TB treatment are excluded in PR. Worsening of radiological findings was defined when pleural effusion increased or new parenchymal lesion developed. Two independent radiologists evaluated the radiographic findings in our study.

\section{Statistical analysis}

All tests of significance were two-sided and were conducted using the Statistical Package for the Social Sciences (SPSS) software (version 16.0: SPSS; Chicago, IL, USA). Values are expressed as mean \pm standard deviation. Univariate comparisons between the PR-positive group and the PR-negative group were performed using the Pearson $X^{2}$ test or Fisher Exact test for categorical variables and the Mann-Whitney $U$ test for continuous variables. Multivariate analysis was performed using logistic regression to adjust other variables. A $P$ value $<0.05$ was considered statistically significant.

\section{Patients with pleural TB}

Over the course of our study, 139 patients (80 males and 59 females) were diagnosed as having pleural TB (Table 1). The mean age was 46.6 years. Past medical history of tuberculosis was corresponded to 18 patients $(12.9 \%)$. The 12 patients $(8.6 \%)$ were suffered from diabetes mellitus and 7 patients were chronic alcoholics. Mean treatment duration and follow-up duration were 7.2 months and 14.7 months.

\section{Clinical characteristics of the 32 patients with $P R$}

PR occurred in $32(23 \%)$ patients after they began anti-TB medication (Table 2). These PR patients included 18 males and 14 females with the mean age of 37.9 years. The mean onset time of PR was 51.1 days after starting anti-TB medication. The patients over half had no symptom. Out of 32 patients, 18 patients received additional
Table 1. Demographic and clinical characteristics of 139 patients with pleural tuberculosis.

\begin{tabular}{lc}
\hline \multicolumn{1}{c}{ Characteristic } & $N=139$ \\
\hline Age, years & $46.6 \pm 21.6$ \\
Male gender & $80(57.6 \%)$ \\
Body mass index & $21.2 \pm 3.0$ \\
Active smoker & $44(31.7 \%)$ \\
Past history of tuberculosis & $18(12.9 \%)$ \\
Comorbidity & \\
Diabetes mellitus & $12(8.6 \%)$ \\
Chronic alcoholics & $7(5.0 \%)$ \\
Malignancy & $2(1.4 \%)$ \\
Chronic renal failure & $1(0.7 \%)$ \\
Site of pleural effusion & \\
Right & $55(41.0 \%)$ \\
Left & $77(55.4 \%)$ \\
Both & $5(3.6 \%)$ \\
Treatment duration, months & $7.2 \pm 2.2$ \\
Follow-up duration, months & $14.7 \pm 10.2$ \\
\hline
\end{tabular}

Table 2. Clinical characteristics and outcomes of the 32 patients with paradoxical response.

\begin{tabular}{lc}
\hline \multicolumn{1}{c}{ Characteristics } & $N=32$ \\
\hline Age, years & $37.9 \pm 19.2$ \\
Male gender & $18(56.3 \%)$ \\
Time to onset of PR, days & $51.1 \pm 46.0$ \\
Readmission & $15(46.9 \%)$ \\
Main symptoms & \\
None & $17(53.1 \%)$ \\
Dyspnea & $8(25.0 \%)$ \\
Cough & $3(9.4 \%)$ \\
Chest pain & $2(6.3 \%)$ \\
Fever & $2(6.3 \%)$ \\
Treatments & \\
None & $14(43.6 \%)$ \\
Steroids & $7(21.9 \%)$ \\
Simple drainage & $7(21.9 \%)$ \\
Thoracotomy & $4(12.5 \%)$ \\
Residual pleural thickening & $11(34.4 \%)$ \\
\hline
\end{tabular}

treatment for symptom control and 15 patients $(46.9 \%)$ required readmission. Of the 18 patients who needed additional treatment, 7 patients received simple drainage and 4 patients received thoracotomy and 7 patients received steroids because of dyspnea. Of 7 patients who received systemic steroid with $0.5 \mathrm{mg} / \mathrm{kg}$ of prednisolone, $5(71 \%)$ showed improvement after a median of 14 days (7-28 days) of steroid therapy. The 14 patients who did not receive additional therapy also showed improvement after a median of 28.5 days (9-58 days). Steroid responder showed improvement more quickly than non treatment group $(P=$ $0.03)$. 
Table 3. Comparison of characteristics of patients with and without paradoxical response.

\begin{tabular}{|c|c|c|c|}
\hline & $\begin{array}{c}\text { Patients without PR } \\
\quad n=107\end{array}$ & $\begin{array}{c}\text { Patients with PR } \\
\quad n=32\end{array}$ & $P$ value \\
\hline Age, years & $49.2 \pm 21.7$ & $19.2 \pm 3.4$ & 0.007 \\
\hline Male gender & $62(57.9 \%)$ & $18(56.3 \%)$ & NS \\
\hline Body mass index & $21.1 \pm 2.8$ & $21.8 \pm 3.5$ & NS \\
\hline Comorbidity & $13(12.1 \%)$ & $2(6.2 \%)$ & NS \\
\hline Loculated pleural effusion & $24(22.4 \%)$ & $5(16.1 \%)$ & NS \\
\hline Site of effusion (right/left/both) (\%) & $38.3 / 57.0 / 4.7$ & $50.0 / 50.0 / 0$ & NS \\
\hline \multicolumn{4}{|l|}{ Presence of adverse drug reaction } \\
\hline Skin reactions & $11(10.3 \%)$ & $8(25.0 \%)$ & 0.033 \\
\hline Liver function abnormality & $10(9.3 \%)$ & $8(25.0 \%)$ & 0.021 \\
\hline Peripheral neuropathy & $2(1.9 \%)$ & $2(6.3 \%)$ & NS \\
\hline Drug fever & $1(0.9 \%)$ & $2(6.3 \%)$ & NS \\
\hline \multicolumn{4}{|l|}{ Treatment outcome } \\
\hline Duration of anti-TB medication & $7.2 \pm 2.0$ & $7.1 \pm 2.9$ & NS \\
\hline Residual pleural thickening & $34(31.8 \%)$ & $11(36.7 \%)$ & NS \\
\hline
\end{tabular}

NS, not significant.

Table 4. Comparison of laboratory findings of patients with and without paradoxical response.

\begin{tabular}{lccc}
\hline & $\begin{array}{c}\text { Patients without PR } \\
n=107\end{array}$ & $\begin{array}{c}\text { Patients with PR } \\
n=32\end{array}$ & $P$ value \\
\hline Hemoglobin, g/dl & $12.5 \pm 1.7$ & $12.9 \pm 1.5$ & NS \\
WBC count, $/ \mathrm{mm}^{3}$ & $6,476.9 \pm 2,077.7$ & $6,593.8 \pm 1,874.5$ & NS \\
PMN count, $/ \mathrm{mm}^{3}$ & $4,481.1 \pm 1,721.5$ & $4,664.9 \pm 1,710.4$ & NS \\
Lymphocyte count, $/ \mathrm{mm}^{3}$ & $1,167.1 \pm 480.5$ & $1,168.3 \pm 501.2$ & NS \\
Protein, g/dl & $6.5 \pm 0.7$ & $6.8 \pm 0.7$ & 0.028 \\
Albumin, g/dl & $3.4 \pm 0.5$ & $3.7 \pm 0.4$ & $<0.001$ \\
Cholesterol, $\mathrm{mg} / \mathrm{dl}$ & $144.5 \pm 31.8$ & $138.4 \pm 32.2$ & NS \\
LDH, IU/L & $267.2 \pm 183.4$ & $236.5 \pm 103.8$ & NS \\
Pleural effusion & & & 0.001 \\
WBC count, $/ \mathrm{mm}^{3}$ & $3,268.1 \pm 5,753.4$ & $4,466.4 \pm 3,087.6$ & 0.038 \\
PMN \% & $15.0 \pm 21.5$ & $23.5 \pm 24.4$ & 0.040 \\
Lymphocyte $\%$ & $83.7 \pm 22.3$ & $75.3 \pm 25.4$ & NS \\
Protein, g/dl & $1.3 \pm 6.8$ & $0.9 \pm 2.9$ & NS \\
LDH, IU/L & $740.7 \pm 490.0$ & $731.3 \pm 426.2$ & NS \\
ADA, IU/L & $88.1 \pm 23.4$ & $86.7 \pm 23.3$ & \\
\hline
\end{tabular}

NS, not significant.

\section{Comparison of characteristics of patients with and without} PR

Compared with the 107 patients without paradoxical deterioration after receiving anti-TB medication, $\mathrm{PR}$ patients had no differences in sex, body mass index (BMI) and underlying comorbidities, such as diabetes mellitus or malignancy (Table 3). Patients with PR were younger $(P=$ $0.007)$, and had a higher incidence of adverse reactions to anti-TB medication such as drug hypersensitivity reaction of skin $(P=0.033$; Odds ratio $(\mathrm{OR})=2.909$, confidence interval $[\mathrm{CI}], 1.055-8.024)$ and liver function abnormality $(P=0.033$; OR $=3.233$, CI, 1.153-9.070) compared with patients without PR. There was no difference in duration of
anti-TB medication. After treatment, 11 (36.7\%) showed residual pleural thickening and $34(31.8 \%)$ patients showed in the non-PR group $(P=0.783)$.

\section{Risk factors for development of $P R$}

PR patients had no differences in hemoglobin, WBC count and lymphocyte count with patients without PR (Table 4). Patients with PR had higher baseline serum protein levels $(P=0.028)$ and high albumin level $(P<0.001)$. This difference in albumin level was maintained after adjusted by age, sex and BMI $(P=0.010)$. In analysis of pleural effusion, WBC count, proportion of polymorphonuclear cell (PMN) and proportion of lymphocyte was statisti- 


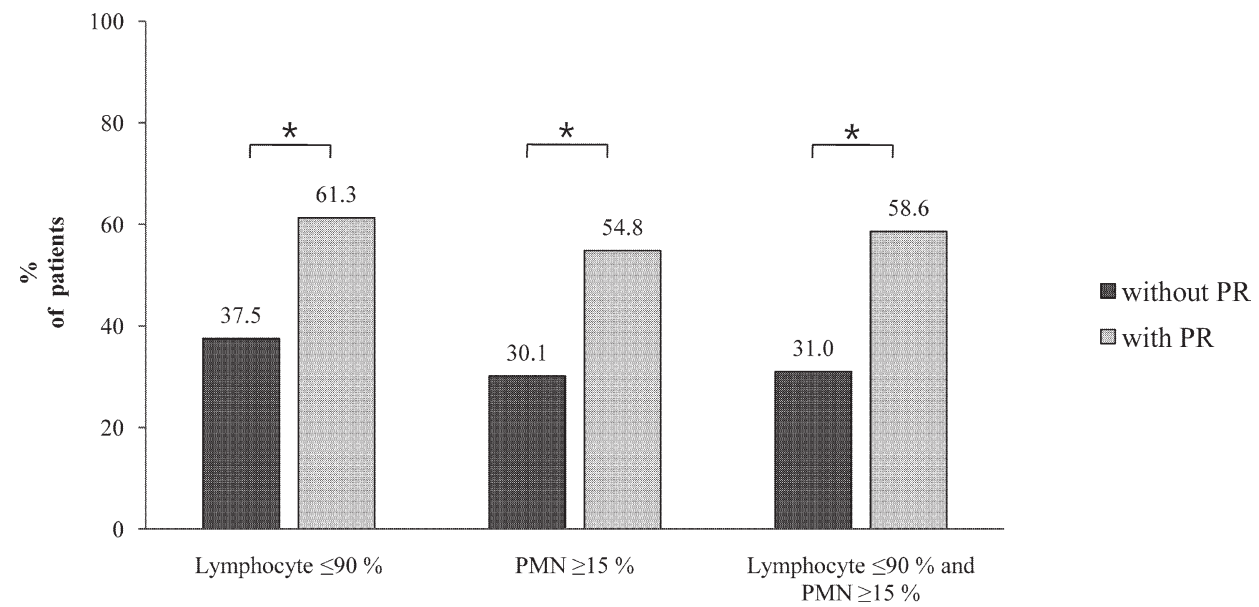

Fig. 1. Comparison of cell proportion of effusion in patients with and without paradoxical response. The PR group had higher percents of patients present the proportion of lymphocyte in inflammatory cells of pleural effusion $\leq 90 \%(P=$ $0.021 ; \mathrm{OR}=2.639, \mathrm{CI}, 1.157-6.019), \mathrm{PMN} \geq 15 \%(P=0.014 ; \mathrm{OR}=2.820, \mathrm{CI}, 1.238-6.424)$ and lymphocyte $\leq 90 \%$ and $\mathrm{PMN} \geq 15 \%(P=0.008 ; \mathrm{OR}=3.153, \mathrm{CI}, 1.345-7.391)$ compared those of non PR group. $* P<0.05$

cally difference between PR negative group and PR positive group $(P<0.05)$.

Especially, the risk factors for PR were the proportion of lymphocyte in inflammatory cells of pleural effusion $\leq 90 \%(P=0.021 ; \mathrm{OR}=2.639, \mathrm{CI}, 1.157-6.019)$ and the proportion of $\mathrm{PMN} \geq 15 \%(P=0.014 ; \mathrm{OR}=2.820, \mathrm{CI}$, 1.238-6.424) (Fig. 1). The OR was 3.153 (CI, 1.345-7.391) with lymphocyte $\leq 90 \%$ and $\mathrm{PMN} \geq 15 \%(P=0.008)$.

\section{Discussion}

In our study, PR was present in $32(23 \%)$ of 139 patients after a mean 51.1 days following initiation of treatment. The 18 patients $(56.4 \%)$ with PR needed additional treatment such as simple drainage, thoracotomy and steroids for symptom control. PR patients had a high incidence of adverse drug reaction such as drug skin reaction or liver function abnormality as compared with patients without PR. The risk factors for PR were younger age, high serum albumin level, low proportion of lymphocyte, and high proportion of PMN in pleural fluid.

Modern chemotherapy regimens for the treatment of TB have a low rate of failure. Excellent results are also achieved when treating pleural TB (Dutt et al. 1986). However, tuberculosis can worsen despite effective chemotherapy; this troublesome condition is termed PR. PR during TB treatment has been explored mainly in HIV-infected patients when beginning highly active antiretroviral therapy (Chambers et al. 1984; Wendel et al. 2001; Navas et al. 2002; Breen et al. 2004). The development of HAARTassociated immunity leads to a variety of new clinical manifestations, due to an increased CD4 T cell count in HIVpositive patients (DeSimone et al. 2000). The mechanism of this PR has been postulated to be an interaction between the host's immune response and the direct effects of mycobacterial products (Afghani and Lieberman 1994).

PR has been reported in HIV-negative patients (Cheng et al. 2002; Breen et al. 2004; Cho et al. 2009). However, the incidence of PR is variable. Chen et al. found that only $2.4 \%$ of pulmonary TB patients developed PR (Cheng et al. 2007). However, a recent study reported that $23 \%$ of patients who had lymph-node TB had PR (Cho et al. 2009). We believe these differences may be due to the site of infection. In the present study, the incidence of PR in pleural TB with HIV-negative patients was 23\% (32 of 137), which was consistent with a previous report on lymph-node TB (Cho et al. 2009). Therefore, the present study shows that PR was not an uncommon phenomenon in pleural TB with HIV-negative patients.

PR is usually a mild, transient and self-limited phenomenon. The mainstay of PR management is the continuation of anti-TB treatment without any modification of the drug regimen. However, PR may deserve special attention in pleural TB as respiratory function can be compromised in cases with massive pleural effusion. These patients often require additional treatments. In our cases, 7 patients received simple drainage of pleural fluid, 4 patients, thoracotomy and 7 patients, steroid medication. Steroid treatment has been used in the management of PR. Some case reports have described rapid recovery after initiation of corticosteroid therapy (Chambers et al. 1984; Hejazi and Hassler 1997). But, it did not show advantage in treating PR in patients with lymph node TB (Cho et al. 2009). In our series, 7 patients received systemic steroids. And the patients with steroids had shorter duration in improvement of pleural effusion compared with the patients who did not receive additional therapy $(P=0.03)$. Therefore, steroids could be used depending on the symptom.

Limited information about risk factors for PR is available in HIV-negative patients with pleural TB. Cheng et al. found that baseline anemia, hypoalbuminemia, lymphopenia, and a greater change in lymphocyte count were independent risk factors for developing PR in pulmonary TB 
(Cheng et al. 2007). Another study reported that risk factors for developing PR in culture positive TB infection are lower baseline lymphocyte counts and a greater surge in lymphocyte counts during PR (Cheng et al. 2003). In contrast, Cho et al. found that younger age and male gender were risk factors for PR in lymph node TB. Our data showed that younger age and a high baseline albumin level were risk factors for PR in pleural TB. However, baseline anemia and lymphopenia were not risk factors for PR. The new finding of our study was that the presence of skin reaction or liver enzyme abnormality was related with PR. Drug hypersensitivity such as urticaria and rash is immune cell mediated hypersensitivity reaction to medication, and anti-TB drug-induced hepatotoxicity is an idiosyncratic reaction to drug metabolites by $\mathrm{T}$ cells (Tostmann et al. 2008). These mechanisms may be similar to that of PR. In active tuberculosis, mycobacterial protein-induced TGF- $\beta$ in monocytes may be responsible for the well-established immunosuppressive response (Toossi et al. 1995). When anti-TB chemotherapy treatment begins, T-cell responses are gradually restored and cytokine levels (such as IFN- $\gamma$ and TNF- $\alpha$ ) normalize. The associated decline of live bacterial load and increase in components of dead mycobacteria may result in an increase in priming of monocytes $/ \mathrm{mac}-$ rophages in situ and an enhancement of the production of inflammatory cytokines, such as TNF- $\alpha$. Therefore, radiological and clinical worsening of a previous lesion or the development of a new lesion may occur after initial improvement during the process of antituberculous treatment (Marshall and Chambers 1998). In a series of this response, a mixed type-1 $\mathrm{T}$ helper cell and type- $2 \mathrm{~T}$ helper cell response were found to be related (Grange et al. 1995). Pleural TB is thought to result from rupture of a subpleural pulmonary tuberculous focus into the pleural space, which allows tuberculoproteins to enter the pleural space and generate the hypersensitive reaction responsible for most of the clinical manifestations (Berger and Mejia 1973). Therefore, the presence of adverse drug reactions might be more common in the PR group.

In our study, the risk factors for PR were the proportion of lymphocyte in inflammatory cells of pleural effusion $\leq 90 \%$ or the proportion of $\mathrm{PMN} \geq 15 \%$. In general, the fluid of TB pleurisy is an exudate, with lymphocytic predominance in about $90 \%$ of cases (Lin et al. 2009). In several study, the initial stage of illness, the differential cell count may reveal polymorphonuclear cell predominance with a subsequent change to a lymphocytic predominance within 1 week is seen (Batungwanayo et al. 1993; Lin et al. 2009). In light of these, it is supposed that ant-TB medication in early neutrophilic inflammation phase may trigger the restoration of immune response, and as a results, PR in pleural TB is induced. There was no study that investigated the condition of pleural effusion to evaluate the risk factors for PR in pleural TB. This study is the first study about the risk factors for PR in pleural TB.

Our study had limitations. Of 139 patients, only 28 patients were culture-positive for $M$. tuberculosis. Therefore, several patients could not be evaluated using the drug susceptibility test. In South Korea, the rate of multidrug-resistant TB among newly diagnosed cases in 2003 was $2.4 \%$, and the resistance to at least one drug was $12.8 \%$ (Kim et al. 2010). Based on these findings, some PR patients in our study may have had drug-resistant TB. However, all of the patients who showed PR improved after 7 to 58 days after initiation of PR. Therefore, the possibility of drug resistance was low. Second, our study is limited by its retrospective nature and relatively small sample size. However, few data are available for the PR in pleural TB with negative of HIV. Therefore, our data might be meaningful.

In conclusion, paradoxical response was not an uncommon problem in non-HIV-infected patients with pleural TB, and half of whom needed additional treatment. Therefore, physicians must pay more attention for PR during the management of pleural TB in the expected patients to develop PR.

\section{Acknowledgments}

This research was supported by the Chung-Ang University Research Grants in 2010.

\section{Conflict of Interest}

The authors report no conflict of interest.

\section{References}

Afghani, B. \& Lieberman, J.M. (1994) Paradoxical enlargement or development of intracranial tuberculomas during therapy: case report and review. Clin. Infect. Dis., 19, 1092-1099.

Al-Majed, S.A. (1996) Study of paradoxical response to chemotherapy in tuberculous pleural effusion. Respir. Med., 90, 211-214.

Bass, J.B. Jr., Farer, L.S., Hopewell, P.C., O’Brien, R., Jacobs, R.F., Ruben, F., Snider, D.E. Jr. \& Thornton, G. (1994) Treatment of tuberculosis and tuberculosis infection in adults and children. American Thoracic Society and The Centers for Disease Control and Prevention. Am. J. Respir. Crit. Care Med., 149, 1359-1374.

Batungwanayo, J., Taelman, H., Allen, S., Bogaerts, J., Kagame, A. \& Van de Perre, P. (1993) Pleural effusion, tuberculosis and HIV-1 infection in Kigali, Rwanda. AIDS, 7, 73-79.

Baumann, M.H., Nolan, R., Petrini, M., Lee, Y.C., Light, R.W. \& Schneider, E. (2007) Pleural tuberculosis in the United States: incidence and drug resistance. Chest, 131, 1125-1132.

Berger, H.W. \& Mejia, E. (1973) Tuberculous pleurisy. Chest, 63, 88-92.

Breen, R.A., Smith, C.J., Bettinson, H., Dart, S., Bannister, B., Johnson, M.A. \& Lipman, M.C. (2004) Paradoxical reactions during tuberculosis treatment in patients with and without HIV co-infection. Thorax, 59, 704-707.

Chambers, S.T., Hendrickse, W.A., Record, C., Rudge, P. \& Smith, H. (1984) Paradoxical expansion of intracranial tuberculomas during chemotherapy. Lancet, 2, 181-184.

Cheng, S.L., Wang, H.C. \& Yang, P.C. (2007) Paradoxical response during anti-tuberculosis treatment in HIV-negative patients with pulmonary tuberculosis. Int. J. Tuberc. Lung Dis., 11, 1290-1295.

Cheng, V.C., Ho, P.L., Lee, R.A., Chan, K.S., Chan, K.K., Woo, P.C., Lau, S.K. \& Yuen, K.Y. (2002) Clinical spectrum of 
paradoxical deterioration during antituberculosis therapy in non-HIV-infected patients. Eur. J. Clin. Microbiol. Infect. Dis., 21, 803-809.

Cheng, V.C., Yam, W.C., Woo, P.C., Lau, S.K., Hung, I.F., Wong, S.P., Cheung, W.C. \& Yuen, K.Y. (2003) Risk factors for development of paradoxical response during antituberculosis therapy in HIV-negative patients. Eur. J. Clin. Microbiol. Infect. Dis., 22, 597-602.

Cho, O.H., Park, K.H., Kim, T., Song, E.H., Jang, E.Y., Lee, E.J., Chong, Y.P., Choi, S.H., Lee, S.O., Woo, J.H., Kim, Y.S. \& Kim, S.H. (2009) Paradoxical responses in non-HIV-infected patients with peripheral lymph node tuberculosis. J. Infect., 59, 56-61.

Choi, Y.W., Jeon, S.C., Seo, H.S., Park, C.K., Park, S.S., Hahm, C.K. \& Joo, K.B. (2002) Tuberculous pleural effusion: new pulmonary lesions during treatment. Radiology, 224, 493-502.

DeSimone, J.A., Pomerantz, R.J. \& Babinchak, T.J. (2000) Inflammatory reactions in HIV-1-infected persons after initiation of highly active antiretroviral therapy. Ann. Intern. Med., 133, 447-454.

Dutt, A.K., Moers, D. \& Stead, W.W. (1986) Short-course chemotherapy for pleural tuberculosis. Nine years' experience in routine treatment service. Chest, 90, 112-116.

Ferrer Sancho, J. (1996) Pleural tuberculosis: incidence, pathogenesis, diagnosis \& treatment. Curr. Opin. Pulm. Med., 2, 327-334.

Gopi, A., Madhavan, S.M., Sharma, S.K. \& Sahn, S.A. (2007) Diagnosis and treatment of tuberculous pleural effusion in 2006. Chest, 131, 880-889.

Grange, J.M., Stanford, J.L. \& Rook, G.A. (1995) Tuberculosis and cancer: parallels in host responses and therapeutic approaches? Lancet, 345, 1350-1352.

Hejazi, N. \& Hassler, W. (1997) Multiple intracranial tuberculomas with atypical response to tuberculostatic chemotherapy: literature review and a case report. Infection, 25, 233-239.

Kim, D.H., Kim, H.J., Park, S.K., Kong, S.J., Kim, Y.S., Kim, T.H., Kim, E.K., Lee, K.M., Lee, S.S., Park, J.S., Koh, W.J., Lee, C.H. \& Shim, T.S. (2010) Treatment outcomes and survival based on drug resistance patterns in multidrug-resistant tuberculosis. Am. J. Respir. Crit. Care Med., 182, 113-119.

Kim, H.J., Lee, H.J., Kwon, S.Y., Yoon, H.I., Chung, H.S., Lee, C.T., Han, S.K., Shim, Y.S. \& Yim, J.J. (2006) The prevalence of pulmonary parenchymal tuberculosis in patients with tuberculous pleuritis. Chest, 129, 1253-1258.

Kumar, R. \& Vasu, T.S. (2001) Paradoxical response to chemotherapy in tuberculous pleural effusion. Saudi. Med. J., 22, 812-813.

Lin, M.T., Wang, J.Y., Yu, C.J., Lee, L.N. \& Yang, P.C. (2009) Mycobacterium tuberculosis and polymorphonuclear pleural effusion: incidence and clinical pointers. Respir. Med., 103, 820-826.

Marshall, B.G. \& Chambers, M.A. (1998) Central nervous system tuberculosis-the paradox of the host immune response. $J$. Infect., 36, 3-4.

Navas, E., Martin-Davila, P., Moreno, L., Pintado, V., Casado, J.L., Fortun, J., Perez-Elias, M.J., Gomez-Mampaso, E. \& Moreno, S. (2002) Paradoxical reactions of tuberculosis in patients with the acquired immunodeficiency syndrome who are treated with highly active antiretroviral therapy. Arch. Intern. Med., 162, 97-99.

Seibert, A.F., Haynes, J. Jr., Middleton, R. \& Bass, J.B. Jr. (1991) Tuberculous pleural effusion. Twenty-year experience. Chest, 99, 883-886.

Smith, H. (1987) Paradoxical responses during the chemotherapy of tuberculosis. J. Infect., 15, 1-3.

Toossi, Z., Young, T.G., Averill, L.E., Hamilton, B.D., Shiratsuchi, H. \& Ellner, J.J. (1995) Induction of transforming growth factor beta 1 by purified protein derivative of Mycobacterium tuberculosis. Infect. Immun., 63, 224-228.

Tostmann, A., Boeree, M.J., Aarnoutse, R.E., de Lange, W.C., van der Ven, A.J. \& Dekhuijzen, R. (2008) Antituberculosis druginduced hepatotoxicity: concise up-to-date review. J. Gastroenterol. Hepatol., 23, 192-202.

Wendel, K.A., Alwood, K.S., Gachuhi, R., Chaisson, R.E., Bishai, W.R. \& Sterling, T.R. (2001) Paradoxical worsening of tuberculosis in HIV-infected persons. Chest, 120, 193-197. 